\title{
MONTE CARLO COMPARISON OF THE METHODS FOR ESTIMATING THE WEIBULL DISTRIBUTION PARAMETERS - WIND SPEED APPLICATION
}

Wind energy is considered one of the most significant alternative energy sources for electrical energy production. The most important factor for modelling wind energy is the wind speed. The two-parameter Weibull distribution is commonly used for modelling wind speed. Its parameters play an important role in the wind energy applications and so it is important to choose the best method for the estimation of these parameters. In this paper we investigate and compare six different methods for estimating the Weibull distribution parameters. The methods we considered are the maximum likelihood method, the method of moments, the empirical method, the power density method, the least squares method and the weighted least squares method. The performance of these methods is compared and discussed through the Monte Carlo simulation.

Keywords: Wind speed, Weibull distribution, parameter estimation, Monte Carlo simulation.

\section{Introduction}

Wind energy represents a form of solar energy that appears due to uneven heating of the Earth. Approximately 1- $2 \%$ of energy emitted by the Sun is transformed to wind energy. This kind of energy has been used for centuries as a drive for wind mills, water pumps or for ships. The interest in the wind energy has been regained recently because of its high potential in electrical energy production. It is motivated by the efforts to lessen the pollution and its negative impacts on the global environment since one of the major greenhouse gases producers are electric power plants through use of fossil fuels. On the other hand, wind provides a renewable, sustainable and environmentally friendly energy source.

The most important parameter for modelling wind energy is the wind speed. It is a random variable changing noticeably in time and space and is influenced by local climatic conditions and terrain [1 -2]. Thus, for wind energy applications it is necessary to describe variation of wind speed using statistical methods.

In the literature several probability distributions (e.g. lognormal, Rayleigh, Weibull, gamma, normal) have been studied to describe the wind speed [3 - 6] For applications in wind energy studies the two parameter Weibull distribution is frequently used in modelling the wind speed distributions [4, 7 - 8] It is due to its easy implementation and flexibility.

There are several methods for estimating the Weibull distribution parameters $[5,7,9-12]$. In recent years these methods have been compared several times and in different ways [13 - 17]. Since the Weibull distribution parameters play an important role in the wind speed applications, it is essential to compare a variety of methods for estimation of the Weibull distribution parameters in order to find the best fitting one. The suitability of the method may vary with the sample size, sample data distribution, sample data format and goodness of fit test [12].

In this paper we study six methods, namely, maximum likelihood method (MLM), the method of moments (MOM), the empirical method (EM), the empirical method of Lysen (EML), the power density method (PDM), the least squares method (LSM) and the weighted least squares method (WLSM). The performances of these methods are compared and discussed through the Monte Carlo simulation. The values of the parameters that are frequently occurring in the wind speed applications are chosen in simulation. The methods are compared using bias and the root mean square error (RMSE). All computations for simulation and parameter estimation are done using Matlab.

\footnotetext{
* ${ }^{1}$ Ivana Pobocikova, ${ }^{1}$ Zuzana Sedliackova, ${ }^{1}$ Maria Michalkova, ${ }^{2}$ Florence George

${ }^{1}$ Department of Applied Mathematics, Faculty of Mechanical Engineering, University of Zilina, Slovakia

${ }^{2}$ Department of Mathematics \& Statistics, Florida International University, Miami, USA

E-mail: ivana.pobocikova@fstroj.uniza.sk
} 


\section{The Weibull distribution}

The Weibull distribution is named after Waloddi Weibull who described it in detail in 1951 . The probability density function $f(x)$ and the cumulative distribution function $F(x)$ of the twoparameter Weibull distribution are given by

$f(x)=\frac{k}{c^{k}} x^{x-1} \exp \left(-\left(\frac{x}{c}\right)^{k}\right)$

and

$F(x)=1-\exp \left(-\left(\frac{x}{c}\right)^{k}\right)$

respectively, for $x>0, k>0$ and $c>0$. Here $x$ is the wind speed, $k$ is the dimensionless shape parameter and $c$ is the scale parameter in units of the wind speed.

The mean $E(X)$ and the variance $D(X)$ of the Weibull distribution are given by

$$
\begin{aligned}
& E(X)=c \Gamma\left(1+\frac{1}{k}\right), \\
& D(X)=c^{2}\left[\Gamma\left(1+\frac{2}{k}\right)-\Gamma^{2}\left(1+\frac{1}{k}\right)\right]
\end{aligned}
$$

respectively, where $\Gamma(a)$ is the gamma function defined by

$\Gamma(a)=\int_{0}^{\infty} x^{a-1} e^{-x} d x, a>0$.

It is known that the Weibull shape parameter $k$ generally ranges from 1.5 to 3 for most wind speed conditions in the world [1]. The Weibull distribution is right skewed, reflecting the fact that the strong winds are rare while the moderate and fresh winds are more common. The higher value of the shape parameter $k$ indicates more stability in the wind speed while the higher value of the scale parameter $c$ indicates that the wind speed is higher.

\section{Methods for estimating the Weibull distribution parameters}

In this section we give a brief description of the methods that will be used for estimation of the Weibull distribution parameters.

Let $X_{1}, X_{2}, \ldots, X_{n}$ be a random sample of size $n$ from the Weibull distribution with the parameters $k$ and $c$. Let $x_{1}, x_{2}, \ldots, x_{n}$ be a realization of a random sample. Now let $X_{(1)}<X_{(2)}<\ldots X_{(n)}$ be the ordered statistics of $X_{1}, X_{2}, \ldots, X_{n}$ and let $x_{(1)}, x_{(2)}, \ldots, X_{(n)}$ be ordered observations. The estimates of the parameters $k$ and $c$ we denote $\hat{k}$ and $\hat{c}$, respectively.

\subsection{Maximum likelihood method}

The likelihood function of the Weibull distribution is given by

$L(k, c)=\prod_{i=1}^{n} \frac{k}{c^{k}} x_{i}^{k-1} \exp \left(-\left(\frac{x_{i}}{c}\right)^{k}\right)$.

By differentiating the logarithm of the function (2) with respect to $k$ and $c$, respectively, and equating them to zero, we obtain

$$
\begin{aligned}
& \frac{\partial \ln L(k, c)}{\partial k}=\frac{n}{k}-n \ln c-\frac{\sum_{i=1}^{n} x_{i}^{k} \ln x_{i}-\ln c \sum_{i=1}^{n} x_{i}^{k}}{c^{k}}+ \\
& +\sum_{i=1}^{n} \ln x_{i}=0, \\
& \frac{\partial \ln L(k, c)}{\partial c}=-\frac{n k}{c}+\frac{k}{c^{k+1}} \sum_{i=1}^{n} x_{i}^{k}=0 .
\end{aligned}
$$

The shape parameter $k$ and the scale parameter $c$ can be estimated by following equations [3]

$$
\begin{aligned}
& \frac{1}{k}-\frac{\sum_{i=1}^{n} x_{i}^{k} \ln x_{i}}{\sum_{i=1}^{n} x_{i}^{k}}+\frac{1}{n} \sum_{i=1}^{n} \ln x_{i}=0 \\
& c=\left(\frac{1}{n} \sum_{i=1}^{n} x_{i}^{k}\right)^{l / k}
\end{aligned}
$$

The estimate $\hat{k}$ of the parameter $k$ can be obtained by solving (3) with respect to $k$. The estimate $\hat{c}$ of the parameter $c$ can be obtained using equation (4). The Newton method was used for the numerical computations.

\subsection{Method of moments}

The estimates of the parameters $k$ and $c$ can be obtained by equating the moments of the Weibull distribution with the corresponding sample moments. The shape parameter $k$ and the scale parameter $c$ can be estimated by following equations [3]

$c \Gamma\left(1+\frac{1}{k}\right)=\bar{x}$

$c^{2} \Gamma\left(1+\frac{2}{k}\right)=\frac{1}{n} \sum_{i=1}^{n} x_{i}^{2}$

where $\bar{x}=\frac{1}{n} \sum_{i=1}^{n} x_{i}$ is the sample mean wind speed. Dividing (6) with the square of (5) we obtain

$\frac{\Gamma\left(1+\frac{2}{k}\right)}{\Gamma^{2}\left(1+\frac{1}{k}\right)}=\frac{\frac{1}{n} \sum_{i=1}^{n} x_{i}^{2}}{\bar{x}^{2}}$ 
The estimate $\hat{k}$ of the parameter $k$ can be obtained by solving (7) with respect to $k$. This equation does not have an analytical solution and can be solved iteratively with respect to $k$. The estimate $\hat{c}$ of the parameter $c$ can be obtained using equation (5).

\subsection{Empirical method}

The empirical method can be used as a special case of the method of moments. The shape parameter $k$ can be estimated by following equation $[6,12]$

$\hat{k}=\left(\frac{\bar{x}}{S_{x}}\right)^{1.086}$

where $s_{x}=\sqrt{\frac{1}{n-1} \sum_{i=1}^{n}\left(x_{i}-\bar{x}\right)^{2}}$ is the sample standard deviation. The scale parameter $c$ can be estimated using (5).

\subsection{Empirical method of Lysen}

In the empirical method suggested by Lysen [18] the shape parameter $k$ can be estimated using (8) and the scale parameter $c$ can be estimated using

$\hat{c}=\bar{x}\left(0.568+\frac{0.433}{k}\right)^{-1 / k}$.

\subsection{Power density method}

The energy pattern factor is defined as [12]

$E_{p f}=\frac{\overline{x^{3}}}{\bar{x}^{3}}$,

where $\overline{x^{3}}=\frac{1}{n} \sum_{i=1}^{n} x_{i}^{3}$ is the sample mean of the wind speed cubes. The shape parameter $k$ can be estimated by following equation

$\hat{k}=1+\frac{3.69}{\left(E_{p f}\right)^{2}}$,

and the scale parameter $c$ can be estimated using (5).

\subsection{Least squares method}

The cumulative distribution function (1) can be linearized as follows

$\ln (-\ln (1-F(x)))=k \ln (x)-k \ln (c)$.

Let $Y=\ln (-\ln (1-F(x))), X=\ln x, b=k$ and $a=-k \ln c$. Then the equation (9) can be rewritten as
$Y=b X+a$

The parameters in equation (10) can be estimated using the least squares method. The estimates of the regression parameters $a$ and $b$ minimize the function $Q(a, b)=\sum_{i=1}^{n}\left(Y_{i}-a-b \ln x_{(i)}\right)^{2}$.

Then the shape parameter $k$ and the scale parameter $c$ can be estimated by following equations

$$
\begin{aligned}
& \hat{k}=\frac{n \sum_{i=1}^{n} \ln x_{(i)} \ln \left[-\ln \left(1-\hat{F}\left(x_{(i)}\right)\right)\right]}{n \sum_{i=1}^{n} \ln ^{2} x_{(i)}-\left(\sum_{i=1}^{n} \ln x_{(i)}\right)^{2}}- \\
& -\frac{\sum_{i=1}^{n} \ln x_{(i)} \sum_{i=1}^{n} \ln \left[-\ln \left(1-\hat{F}\left(x_{(i)}\right)\right)\right]}{n \sum_{i=1}^{n} \ln ^{2} x_{(i)}-\left(\sum_{i=1}^{n} \ln x_{(i)}\right)^{2}} \\
& \hat{c}=\exp \left(-\frac{\sum_{i=1}^{n} \ln \left[-\ln \left(1-\hat{F}\left(x_{(i)}\right)\right)\right]-\hat{k} \sum_{i=1}^{n} \ln x_{(i)}}{\hat{k} n}\right) .
\end{aligned}
$$

Based on our previous study [19] to estimate the values of the cumulative distribution function $F(x)$ the mean rank is used $\hat{F}\left(x_{(i)}\right)=\frac{i}{n+1}$,

where $i$ denotes the $i^{\text {th }}$ smallest value of $x_{(1)}, x_{(2)}, \ldots, x_{(n)}$, $i=1,2, \ldots, n$.

\subsection{Weighted least squares method}

The estimates of the regression parameters $a$ and $b$ minimize the function

$Q(a, b)=\sum_{i=1}^{n} w_{i}\left(Y_{i}-a-b \ln x_{(i)}\right)^{2}$

where $w_{i}$ is the weight factor, $i=1,2, \ldots, n$. In this paper we use the weight factor proposed in [20]

$w_{i}=\left[\left(1-\hat{F}\left(x_{(i)}\right)\right) \ln \left(1-\hat{F}\left(x_{(i)}\right)\right)\right]^{2}, i=1,2, \ldots, n$.

The shape parameter $k$ and the scale parameter $c$ can be estimated by following equations

$\hat{k}=\frac{\sum_{i=1}^{n} w_{i} \sum_{i=1}^{n} w_{i} \ln x_{(i)} \ln \left[-\ln \left(1-\hat{F}\left(x_{(i)}\right)\right)\right]}{\sum_{i=1}^{n} w_{i} \sum_{i=1}^{n} w_{i} \ln ^{2} x_{(i)}-\left(\sum_{i=1}^{n} w_{i} \ln x_{(i)}\right)^{2}}-$
$-\frac{\sum_{i=1}^{n} w_{i} \ln x_{(i)} \sum_{i=1}^{n} w_{i} \ln \left[-\ln \left(1-\hat{F}\left(x_{(i)}\right)\right)\right]}{\sum_{i=1}^{n} w_{i} \sum_{i=1}^{n} w_{i} \ln ^{2} x_{(i)}-\left(\sum_{i=1}^{n} w_{i} \ln x_{(i)}\right)^{2}}$, 
$\hat{c}=\exp \left(-\frac{\sum_{i=1}^{n} w_{i} \ln \left[-\ln \left(1-\hat{F}\left(x_{(i)}\right)\right)\right]-\hat{k} \sum_{i=1}^{n} w_{i} \ln x_{(i)}}{\hat{k} \sum_{i=1}^{n} w_{i}}\right)$.

\section{Simulation study}

A simulation study is conducted to compare the performance of the aforementioned methods for estimating the Weibull distribution parameters.

In simulation, we choose parameter values which appear frequently in the wind speed applications. We consider $k=1.5,2$, 2.5, $c=1$ and sample sizes $n=30,100,500,1000$ in order to cover small, medium and large sample sizes. For each combination $k, c$ and $n$, we generate by the Monte Carlo simulation, $N=5000$ random samples from the Weibull distribution using the inverting the cumulative distribution function (1)

$X=c\left[\ln \left(\frac{1}{1-U}\right)\right]^{1 / k}$,

where $U$ has uniform distribution over the interval $(0,1)$.

For each method discussed, we obtain 5000 estimates of the parameter $k$ and 5000 estimates of the parameter $c$. Then we compute the sample means and the sample variances

$$
\begin{aligned}
\bar{k} & =\frac{1}{5000} \sum_{i=1}^{5000} \hat{k}_{i}, \bar{c}=\frac{1}{5000} \sum_{i=1}^{5000} \hat{c}_{i}, \\
s_{k}^{2} & =\frac{1}{4999} \sum_{i=1}^{5000}\left(\hat{k}_{i}-\bar{k}\right)^{2}, s_{k}^{2}=\frac{1}{4999} \sum_{i=1}^{5000}\left(\hat{c}_{i}-\bar{c}\right)^{2},
\end{aligned}
$$

respectively. For comparing the performance of the methods we consider the bias given by

$\operatorname{bias}(\hat{k})=\bar{k}-k, \operatorname{bias}(\hat{c})=\bar{c}-c$,

respectively, and the sample root mean square error (RMSE) given by

$$
\begin{aligned}
& \operatorname{RMSE}(\hat{k})=\sqrt{\frac{1}{5000} \sum_{i=1}^{5000}\left(\hat{k}_{i}-k\right)^{2}}, \\
& \operatorname{RMSE}(\hat{c})=\sqrt{\frac{1}{5000} \sum_{i=1}^{5000}\left(\hat{c}_{i}-c\right)^{2}},
\end{aligned}
$$

respectively. Ideal value of $R M S E$ is close to zero. The methods with smaller RMSE are preferred. When RMSE of two methods are close to each other, the method with less bias will be preferred.

\section{Results and discussion}

The results of the Monte Carlo simulation are presented in Tables $1-3$. From the simulation results, we observe the following with regards to bias and RMSE of the two parameters:

- Bias for the shape parameter $k$ : It is observed that MLM and MOM have the smallest biases in majority of cases except the one when $k=2.5, n=30,100$. In this case PDM performs the best. For $k=2, n=30$ both EM and EML outperform other methods.

- Bias for the scale parameter $c$ : It is observed that MLM, MOM and PDM become the best methods except those cases when $k=1.5, n=30$ and $k=2$ and $n=30,100$. Here EML performs the best.

- $R M S E$ for the shape parameter $k$ : It is observed that MLM, followed by MOM, has the smallest RMSE for $k=1.5,2$ except the case when $k=2, n=30$. Here EM and EML perform the best. When $k=2.5$, PDM outperforms other methods for all sample sizes, followed by EM and EML for $n=30,100,500$ and MLM for $n=1000$.

- $R M S E$ for the scale parameter $c$ : It is observed that MLM performs the best for $k=1.5,2$ and 2.5 for all sample sizes except the case when $k=2.5, n=500$. Here EML performs the best. Moreover, for parameter $c$ MOM, MLM, EM, EML, PDM and WLSM are comparable methods.

In addition to the above, we have noticed the following from the simulation results:

- It can be seen that the values of the variance, bias and RMSE decrease when the sample size increases, for all seven compared methods.

- LSM has generally the highest biases and RMSE for both parameters compared to other methods. WLSM is the second worst method in terms of RMSE.

- WLSM outperforms LSM in terms of the bias and RMSE.

- It should be noted that there is not much difference in the performance between MLM and MOM, especially when the sample sizes are large.

- EM and EML are comparable methods in terms of bias and RMSE. 
Simulation results, $k=1.5, c=1$

Table 1

\begin{tabular}{|c|c|c|c|c|c|c|c|c|}
\hline Method & $\bar{k}$ & $\bar{c}$ & $S_{k}^{2}$ & $S_{c}^{2}$ & $\operatorname{bias}(\hat{k})$ & $\operatorname{bias}(\hat{c})$ & $R M S E(\hat{k})$ & $R M S E(\hat{c})$ \\
\hline \multicolumn{9}{|l|}{$n=\mathbf{3 0}$} \\
\hline MLM & 1.57482 & 0.99865 & 0.05705 & 0.01651 & 0.07482 & -0.00135 & 0.25027 & 0.12849 \\
\hline MOM & 1.58379 & 0.99910 & 0.05853 & 0.01659 & 0.08379 & -0.00090 & 0.25600 & 0.12878 \\
\hline EM & 1.57623 & 0.99857 & 0.05739 & 0.01661 & 0.07623 & -0.00143 & 0.25137 & 0.12889 \\
\hline EML & 1.57623 & 0.99928 & 0.05739 & 0.01664 & 0.07623 & -0.00072 & 0.25137 & 0.12900 \\
\hline PDM & 1.61126 & 1.00092 & 0.06141 & 0.01649 & 0.11126 & 0.00092 & 0.27161 & 0.12840 \\
\hline LSM & 1.36198 & 1.03158 & 0.06739 & 0.01863 & -0.13802 & 0.03158 & 0.29399 & 0.14009 \\
\hline WLSM & 1.41356 & 1.01472 & 0.05830 & 0.01839 & -0.08644 & 0.01472 & 0.25643 & 0.13641 \\
\hline \multicolumn{9}{|l|}{$n=100$} \\
\hline MLM & 1.52236 & 0.99959 & 0.01459 & 0.00489 & 0.02236 & -0.00041 & 0.12283 & 0.06994 \\
\hline MOM & 1.52596 & 0.99977 & 0.01583 & 0.00494 & 0.02596 & -0.00023 & 0.12846 & 0.07030 \\
\hline EM & 1.53999 & 1.00082 & 0.01608 & 0.00494 & 0.03999 & 0.00082 & 0.13297 & 0.07028 \\
\hline EML & 1.53999 & 1.00161 & 0.01608 & 0.00495 & 0.03999 & 0.00161 & 0.13297 & 0.07036 \\
\hline PDM & 1.53575 & 1.00036 & 0.01749 & 0.00494 & 0.03575 & 0.00036 & 0.13697 & 0.07028 \\
\hline LSM & 1.42643 & 1.01477 & 0.02243 & 0.00546 & -0.07357 & 0.01477 & 0.16686 & 0.07532 \\
\hline WLSM & 1.47470 & 1.00449 & 0.01975 & 0.00545 & -0.02530 & 0.00449 & 0.14276 & 0.07397 \\
\hline \multicolumn{9}{|l|}{$n=500$} \\
\hline MLM & 1.50441 & 0.99977 & 0.00284 & 0.00094 & 0.00441 & -0.00023 & 0.05345 & 0.03058 \\
\hline MOM & 1.50490 & 0.99975 & 0.00317 & 0.00095 & 0.00490 & -0.00025 & 0.05648 & 0.03075 \\
\hline EM & 1.52588 & 1.00138 & 0.00326 & 0.00094 & 0.02588 & 0.00138 & 0.06268 & 0.03075 \\
\hline EML & 1.52588 & 1.00220 & 0.00326 & 0.00095 & 0.02588 & 0.00220 & 0.06268 & 0.03082 \\
\hline PDM & 1.50677 & 0.99984 & 0.00364 & 0.00095 & 0.00677 & -0.00016 & 0.06074 & 0.03082 \\
\hline LSM & 1.47521 & 1.00451 & 0.00499 & 0.00101 & -0.02479 & 0.00451 & 0.07488 & 0.03204 \\
\hline WLSM & 1.49419 & 1.00063 & 0.00402 & 0.00105 & -0.00581 & 0.00063 & 0.06368 & 0.03242 \\
\hline \multicolumn{9}{|l|}{$n=1000$} \\
\hline MLM & 1.50203 & 1.00011 & 0.00136 & 0.00049 & 0.00203 & 0.00011 & 0.03698 & 0.02219 \\
\hline MOM & 1.50236 & 1.00012 & 0.00153 & 0.00050 & 0.00236 & 0.00012 & 0.03924 & 0.02229 \\
\hline EM & 1.52419 & 1.00182 & 0.00158 & 0.00050 & 0.02419 & 0.00182 & 0.04655 & 0.02235 \\
\hline EML & 1.52419 & 1.00264 & 0.00158 & 0.00050 & 0.02419 & 0.00264 & 0.04655 & 0.02245 \\
\hline PDM & 1.50279 & 1.00013 & 0.00179 & 0.00050 & 0.00279 & 0.00013 & 0.04234 & 0.02235 \\
\hline LSM & 1.48415 & 1.00308 & 0.00242 & 0.00052 & -0.01585 & 0.00308 & 0.05169 & 0.02308 \\
\hline WLSM & 1.49721 & 1.00060 & 0.00201 & 0.00055 & -0.00279 & 0.00060 & 0.04489 & 0.02343 \\
\hline
\end{tabular}


Simulation results, $k=2, c=1$

Table 2

\begin{tabular}{|c|c|c|c|c|c|c|c|c|}
\hline Method & $\bar{k}$ & $\bar{c}$ & $S_{k}^{2}$ & $S_{c}^{2}$ & $\operatorname{bias}(\hat{k})$ & $\operatorname{bias}(\hat{c})$ & $R M S E(\hat{k})$ & $R M S E(\hat{c})$ \\
\hline \multicolumn{9}{|l|}{$n=\mathbf{3 0}$} \\
\hline MLM & 2.09713 & 0.99853 & 0.09963 & 0.00942 & 0.09713 & -0.00147 & 0.33022 & 0.09706 \\
\hline MOM & 2.09368 & 0.99814 & 0.09909 & 0.00944 & 0.09368 & -0.00186 & 0.32841 & 0.09715 \\
\hline EM & 2.07540 & 0.99811 & 0.09274 & 0.00945 & 0.07540 & -0.00189 & 0.31369 & 0.09722 \\
\hline EML & 2.07540 & 0.99861 & 0.09274 & 0.00945 & 0.07540 & -0.00139 & 0.31369 & 0.09720 \\
\hline PDM & 2.10456 & 0.99828 & 0.09064 & 0.00944 & 0.10456 & -0.00172 & 0.31868 & 0.09717 \\
\hline LSM & 1.82231 & 1.02214 & 0.11832 & 0.01051 & -0.17769 & 0.02214 & 0.38713 & 0.10485 \\
\hline WLSM & 1.88200 & 1.00932 & 0.10210 & 0.01031 & -0.11800 & 0.00932 & 0.34059 & 0.10198 \\
\hline \multicolumn{9}{|l|}{$n=100$} \\
\hline MLM & 2.02982 & 0.99923 & 0.02594 & 0.00275 & 0.02982 & -0.00077 & 0.16377 & 0.05248 \\
\hline MOM & 2.02979 & 0.99918 & 0.02631 & 0.00277 & 0.02979 & -0.00082 & 0.16489 & 0.05260 \\
\hline EM & 2.04067 & 0.99931 & 0.02537 & 0.00276 & 0.04067 & -0.00069 & 0.16439 & 0.05257 \\
\hline EML & 2.04067 & 0.99984 & 0.02537 & 0.00276 & 0.04067 & -0.00016 & 0.16439 & 0.05255 \\
\hline PDM & 2.04323 & 0.99926 & 0.02703 & 0.00277 & 0.04323 & -0.00074 & 0.16998 & 0.05259 \\
\hline LSM & 1.90190 & 1.01055 & 0.03988 & 0.00305 & -0.09810 & 0.01055 & 0.22247 & 0.05619 \\
\hline WLSM & 1.96626 & 1.00286 & 0.03510 & 0.00306 & -0.03374 & 0.00286 & 0.19035 & 0.05539 \\
\hline \multicolumn{9}{|l|}{$n=500$} \\
\hline MLM & 2.00544 & 0.99992 & 0.00499 & 0.00053 & 0.00544 & -0.00008 & 0.07084 & 0.02301 \\
\hline MOM & 2.00508 & 0.99987 & 0.00509 & 0.00053 & 0.00508 & -0.00013 & 0.07155 & 0.02304 \\
\hline EM & 2.02555 & 1.00005 & 0.00497 & 0.00053 & 0.02555 & 0.00005 & 0.07496 & 0.02302 \\
\hline EML & 2.02555 & 1.00060 & 0.00497 & 0.00053 & 0.02555 & 0.00060 & 0.07496 & 0.02302 \\
\hline PDM & 2.01723 & 0.99996 & 0.00557 & 0.00053 & 0.01723 & -0.00004 & 0.07660 & 0.02304 \\
\hline LSM & 1.96657 & 1.00345 & 0.00880 & 0.00057 & -0.03343 & 0.00345 & 0.09958 & 0.02405 \\
\hline WLSM & 1.99156 & 1.00057 & 0.00703 & 0.00060 & -0.00844 & 0.00057 & 0.08429 & 0.02443 \\
\hline \multicolumn{9}{|l|}{$n=1000$} \\
\hline MLM & 2.00291 & 1.00010 & 0.00246 & 0.00028 & 0.00291 & 0.00010 & 0.04971 & 0.01665 \\
\hline МOM & 2.00279 & 1.00009 & 0.00253 & 0.00028 & 0.00279 & 0.00009 & 0.05037 & 0.01668 \\
\hline EM & 2.02444 & 1.00027 & 0.00247 & 0.00028 & 0.02444 & 0.00027 & 0.05537 & 0.01666 \\
\hline EML & 2.02444 & 1.00082 & 0.00247 & 0.00028 & 0.02444 & 0.00082 & 0.05537 & 0.01668 \\
\hline PDM & 2.01454 & 1.00018 & 0.00277 & 0.00028 & 0.01454 & 0.00018 & 0.05456 & 0.01668 \\
\hline LSM & 1.97942 & 1.00230 & 0.00429 & 0.00030 & -0.02058 & 0.00230 & 0.06865 & 0.01735 \\
\hline WLSM & 1.99644 & 1.00041 & 0.00359 & 0.00031 & -0.00356 & 0.00041 & 0.06001 & 0.01755 \\
\hline
\end{tabular}


Simulation results, $k=2.5, c=1$

Table 3

\begin{tabular}{|c|c|c|c|c|c|c|c|c|}
\hline Method & $\bar{k}$ & $\bar{c}$ & $S_{k}^{2}$ & $S_{c}^{2}$ & $\operatorname{bias}(\hat{k})$ & $\operatorname{bias}(\hat{c})$ & $R M S E(\hat{k})$ & $R M S E(\hat{c})$ \\
\hline \multicolumn{9}{|l|}{$n=\mathbf{3 0}$} \\
\hline MLM & 2.62307 & 0.99835 & 0.15265 & 0.00594 & 0.12307 & -0.00165 & 0.40959 & 0.07707 \\
\hline MOM & 2.61425 & 0.99804 & 0.15092 & 0.00594 & 0.11425 & -0.00196 & 0.40490 & 0.07710 \\
\hline EM & 2.57699 & 0.99845 & 0.13885 & 0.00596 & 0.07699 & -0.00155 & 0.38046 & 0.07722 \\
\hline EML & 2.57699 & 0.99860 & 0.13885 & 0.00595 & 0.07699 & -0.00140 & 0.38046 & 0.07716 \\
\hline PDM & 2.56055 & 0.99887 & 0.09258 & 0.00600 & 0.06055 & -0.00113 & 0.31021 & 0.07743 \\
\hline LSM & 2.27362 & 1.01730 & 0.17871 & 0.00650 & -0.22638 & 0.01730 & 0.47951 & 0.08248 \\
\hline WLSM & 2.35295 & 1.00769 & 0.15804 & 0.00649 & -0.14705 & 0.00769 & 0.42383 & 0.08090 \\
\hline \multicolumn{9}{|l|}{$n=100$} \\
\hline MLM & 2.53321 & 0.99975 & 0.04160 & 0.00177 & 0.03321 & -0.00025 & 0.20662 & 0.04202 \\
\hline MOM & 2.52979 & 0.99962 & 0.04167 & 0.00177 & 0.02979 & -0.00038 & 0.20627 & 0.04207 \\
\hline EM & 2.52897 & 0.99964 & 0.03937 & 0.00177 & 0.02897 & -0.00036 & 0.20051 & 0.04208 \\
\hline EML & 2.52897 & 0.99981 & 0.03937 & 0.00177 & 0.02897 & -0.00019 & 0.20051 & 0.04205 \\
\hline PDM & 2.50393 & 0.99996 & 0.02970 & 0.00178 & 0.00393 & -0.00004 & 0.17237 & 0.04220 \\
\hline LSM & 2.37843 & 1.00832 & 0.06277 & 0.00192 & -0.12157 & 0.00832 & 0.27845 & 0.04461 \\
\hline WLSM & 2.44818 & 1.00282 & 0.05497 & 0.00197 & -0.05182 & 0.00282 & 0.24009 & 0.04447 \\
\hline \multicolumn{9}{|l|}{$n=500$} \\
\hline MLM & 2.50735 & 0.99975 & 0.00788 & 0.00034 & 0.00735 & -0.00025 & 0.08908 & 0.01835 \\
\hline MOM & 2.50665 & 0.99972 & 0.00796 & 0.00034 & 0.00665 & -0.00028 & 0.08944 & 0.01836 \\
\hline EM & 2.51765 & 0.99961 & 0.00759 & 0.00034 & 0.01765 & -0.00039 & 0.08887 & 0.01836 \\
\hline EML & 2.51765 & 0.99978 & 0.00759 & 0.00034 & 0.01765 & -0.00022 & 0.08887 & 0.01834 \\
\hline PDM & 2.48775 & 0.99992 & 0.00594 & 0.00034 & -0.01225 & -0.00008 & 0.07805 & 0.01840 \\
\hline LSM & 2.45868 & 1.00258 & 0.01387 & 0.00036 & -0.04132 & 0.00258 & 0.12480 & 0.01916 \\
\hline WLSM & 2.49032 & 1.00025 & 0.01117 & 0.00038 & -0.00968 & 0.00025 & 0.10613 & 0.01944 \\
\hline \multicolumn{9}{|l|}{$n=1000$} \\
\hline MLM & 2.50339 & 1.00001 & 0.00379 & 0.00018 & 0.00339 & 0.00001 & 0.06163 & 0.01331 \\
\hline МOM & 2.50316 & 1.00000 & 0.00384 & 0.00018 & 0.00316 & 0.00000 & 0.06203 & 0.01332 \\
\hline EM & 2.51564 & 0.99988 & 0.00366 & 0.00018 & 0.01564 & -0.00012 & 0.06251 & 0.01332 \\
\hline EML & 2.51564 & 1.00005 & 0.00366 & 0.00018 & 0.01564 & 0.00005 & 0.06251 & 0.01331 \\
\hline PDM & 2.48526 & 1.00018 & 0.00288 & 0.00018 & -0.01474 & 0.00018 & 0.05562 & 0.01335 \\
\hline LSM & 2.47359 & 1.00178 & 0.00673 & 0.00019 & -0.02641 & 0.00178 & 0.08615 & 0.01382 \\
\hline WLSM & 2.49535 & 1.00030 & 0.00558 & 0.00020 & -0.00465 & 0.00030 & 0.07482 & 0.01406 \\
\hline
\end{tabular}

\section{Conclusions}

In this paper, we compared the performance of the maximum likelihood method (MLM), the method of moments (MOM), the empirical method (EM), the empirical method of Lysen (EML), the power density method (PDM), the least squares method (LSM) and the weighted least squares method (WLSM) for estimation of the Weibull distribution parameters. The comparisons were conducted in terms of the bias and root mean square error (RMSE) using the Monte Carlo simulation study. Based on the simulation results we have concluded that MLM performs better than the other methods discussed in the paper. MOM and PDM also perform well and can be considered as alternative methods for estimating the Weibull distribution parameters.

\section{Acknowledgement}

This paper was supported by the Slovak Grant Agency VEGA through the project No. 1/0812/17. 


\section{References}

[1] AKPINAR, E. K., AKPINAR, S.: Determination of the Wind Energy Potential for Maden, Energy Conversion and Management, 45, 2004, 2901-2914.

[2] Atlas of Renewable Energy Sources in Slovakia (in Slovak), Energetické centrum Bratislava, 2012. ISBN 978-80-969646-8.

[3] CARTA, J. A., RAMIREZ, P., VElasQueZ, S.: A Review of Wind Speed Probability Distributions Used in Wind Energy Analysis Case Studies in The Canary Island. Renewable and Sustainable Energy Reviews, 13, 2009, 933-955.

[4] CELIK, A. N.: A Statistical Analysis of Wind Power Density Based on the Weibull and Rayleigh Models at the Southern Region of Turkey. Renewable Energy, 29, 2003, 593-604.

[5] JUStUS, C. G., HARgraVeS, W. R., MIKHAIL, A., GRABER, D.: Methods for Estimating Wind Speed Frequency Distributions. J. of Applied Meteorology, 17, 1978, 350-353.

[6] MORGAN, M. C., LACKNER, M., VOGEL, R. M., BAISE, L. G.: Probability Distributions for Offshore Wind Speeds. Energy Conversion and Management, 52, 2011, 15-26.

[7] SEGURO, J. V., LAMBERT, T. W.: Modern Estimation of the Parameters of the Weibull Speed Distribution for Wind Energy Analysis. J. of Wind Engineering and Industrial Aerodynamics, 85, 2000, 75-84.

[8] LUN, I. Y. F., LAM, J. C.: A Study of Weibull Parameters Using Long-Term Wind Observation. Renewable Energy, 20, 2000, 145-153.

[9] STEVENS, M. J. M., SMUlderS, P. T.: The Estimation of the Parameters of the Weibull Wind Speed Distribution for Wind Energy Utilization Purposes. Wind Engineering, vol. 3, 2, 1979, 132-45.

[10] DORVLO, A. S. S.: Estimating Wind Speed Distribution. Energy Conversion and Management, 43, 2002, 2311-2318.

[11] WU, D., ZHOU, J., LI, Y.: Methods for Estimating Weibull Parameters for Brittle Materials. J. of Materials Science, 41, 2006, 5630-5638.

[12] AKDAG, S. A., DINLER, A.: A New Method to Estimate Weibull Parameters for Wind Energy Application. Energy Conversion and Management, 50, 2009, 1761-1766.

[13] KANTAR, Y. M., SENOGLU, B.: A Comparative Study for the Location and Scale Parameter of the Weibull Distribution with Given Shape Parameter. Computers \& Geosciences, 34, 2008, 1900-1909.

[14] COSTA ROCHA, P. A., DE SOUSA, R. C., DE ANDRADE, C. F., DA SILVA, M. E. V.: Comparison of Seven Numerical Methods for Determining Weibull Parameters for Wind Energy Generation in the Northeast Region of Brazil. Applied Energy, 89, 2012, 395-400.

[15] CHANG, T. P.: Performance Comparison of Six Numerical Methods in Estimating Weibull Parameters for Wind Energy Application. Applied Energy, 88, 2011, 272-282.

[16] KANTAR, Y. M., KURBAN, M., HOCAOGLU, F. O.: Comparison of Six Different Parameter Estimation Methods in Wind Power Applications. Scientific Research and Essays, vol. 6, 32, 2011, 6594-6604.

[17] GEORGE, F.: A Comparison of Shape and Scale Estimators of the Two-Parameter Weibull Distribution. J. of Modern Applied Statistical Methods, vol. 13, 1, 23-35.

[18] LYSEN, E.H.: Introduction to Wind Energy, The Netherlands: SWD Publication SWD 82-1, 1983.

[19] POBOCIKOVA, I., SEDLIACKOVA, Z.: The Least Square and the Weighted Least Square Methods for Estimating the Weibull Distribution Parameters-A Comparative Study. Communications - Scientific Letters of the University of Zilina, vol. 14, No. 4, 2012, 88-93.

[20] BERGMAN, B.: Estimation of Weibull Parameters Using a Weight Function. J. of Materials Science Letters, 5, 1986, 611-614. 